\title{
Diffractive Cross Sections Implemented in PYTHIA8-MBR vs LHC Results ${ }^{\S}$
}

\author{
Konstantin Goulianos*
}

The Rockefeller University, 1230 York Avenue, New York, NY 10065, USA

\begin{abstract}
We review the predictions of diffractive cross sections implemented in the PYTHIA8-MBR Monte Carlo simulation and compare them to recent LHC results.
\end{abstract}

PACS Number(s): 12.40.Nn, 12.39.St, 13.85.Lg, 13.85.Fb.

Keywords: Diffractive, inelalstic, soft, total.

\section{INTRODUCTION}

Measurements at the LHC have shown that there are sizable disagreements among Monte Carlo (MC) implementations of "soft" processes based on cross sections proposed by various physics models, and that it is not possible to reliably predict all such processes, or even all aspects of a given process, using a single model [1-3]. In the CDF studies of diffraction at the Tevatron, all processes are well modeled by the MBR (Minimum Bias Rockefeller) MC simulation, which is a stand-alone simulation based on a unitarized Regge-theory model, RENORM [4], employing inclusive nucleon parton distribution functions (PDF's) and QCD color factors. The RENORM model was updated in a presentation at EDS-2009 [5] to include a unique unitarization prescription for predicting the total $p p$ cross section at high energies, and that update has been included as an MBR option for simulating diffractive processes in PYTHIA8 since version PYTHIA8.165 [6], to be referred here-forth as PYTHIA8-MBR. In this paper, we briefly review the cross sections [7] implemented in this option of PYTHIA8 and compare them with LHC measurements.

The PYTHIA8-MBR option includes a full simulation of the hadronization of the implemented diffraction dissociation processes: single, double, and central diffraction. In the MBR simulation used at CDF, the hadronization of the final state(s) was based on a data-driven phenomenological model of multiplicities and $p_{t}$ (transverse momentum) distributions calibrated using $\mathrm{S} \bar{p} p \mathrm{~S}$ and Fermilab fixed-target results. Later, the model was successfully tested against Tevatron minimum bias (MB) and diffraction data. However, only $\pi^{ \pm}$ and $\pi^{0}$ particles were produced in the final state, with multiplicities obeying a statistical model of a modified

*Address correspondence to this author at The Rockefeller University, 1230 York Avenue, New York, NY 10065, USA;

Tel:+1.212.3278817; Fax: +1.212.327.7786;

E-mail: dino@rockefeller.edu

${ }^{\S}$ This paper is an essentially identical update of [3], which in itself is a more substantial update of [1].
Gamma distribution function that provided good fits to experimental data [8]. This model could not be used to predict specific-particle final states. In the PYTHIA8-MBR implementation, hadronization is perfor-med by PYTHIA8 tuned to reproduce final-state distribut-ions in agreement with MBR's, with hadronization done in the PYTHIA8 framework. Thus, all final-state particles are now automatically produced, greatly enhancing the horizon of applicability of PYTHIA8-MBR.

\section{CROSS SECTIONS}

The following diffraction dissociation processes are considered in PYTHIA8-MBR:

$\mathrm{SD}$

$$
p p \rightarrow X p \quad \text { SingleDiffraction/Dissociation }
$$

or $\quad p p \rightarrow p Y \quad$ (the other proton survives)

DD $\quad p p \rightarrow X Y \quad$ Double Diffraction/Dissociation

$\mathrm{CD} / \mathrm{DPE} p p \rightarrow p X p \quad$ Central Diffraction /

$$
\text { Double Pomerom Exchange }
$$

The RENORM predictions are expressed as unitarized Regge-theory formulas, in which the unitarization is achieved by a renormalization scheme where the Pomeron (IP) flux is interpreted as the probability for forming a diffractive (non-exponentially suppressed) rapidity gap and thereby its integral over all phase space saturates at the energy where it reaches unity. Differential cross sections are expressed in terms of the IP-trajectory, $\alpha(t)=1+\varepsilon+\alpha^{\prime} t=1.104+0.25\left(\mathrm{GeV}^{-2}\right) \cdot t$, the IP-p coupling, $\beta(t)$, and the ratio of the triple-IP to the $I P$ - $p$ couplings, $\kappa \equiv g(t) / \beta(0)$. For large rapidity gaps, $\Delta y \geq 3$, for which $I P$-exchange dominates, the cross sections may be written as,

$$
\begin{aligned}
& \frac{d^{2} \sigma_{S D}}{d t d \Delta y}=\frac{1}{N_{\text {gap }}(s)}\left[\frac{\beta^{2}(t)}{16 \pi} e^{2[\alpha(t)-1] \Delta y}\right] \cdot\left\{\kappa \beta^{2}(0)\left(\frac{s^{\prime}}{s_{0}}\right)^{\varepsilon}\right\}, \\
& \frac{d^{3} \sigma_{D D}}{d t d \Delta y d y_{0}}=\frac{1}{N_{\text {gap }}(s)}\left[\frac{\kappa \beta^{2}(0)}{16 \pi} e^{2[\alpha(t)-1] \Delta y}\right] \cdot\left\{\kappa \beta^{2}(0)\left(\frac{s^{\prime}}{s_{0}}\right)^{\varepsilon}\right\},
\end{aligned}
$$


$\frac{d^{4} \sigma_{D P E}}{d t_{1} d t_{2} d \Delta y d y_{c}}=\frac{1}{N_{\mathrm{g} a p}(s)}\left[\Pi_{i}\left[\frac{\beta^{2}\left(t_{i}\right)}{16 \pi} e^{2\left[\alpha\left(t_{i}\right)-1\right] \Delta y_{i}}\right]\right] \cdot \kappa\left\{\kappa \beta^{2}(0)\left(\frac{s^{\prime}}{s_{0}}\right)^{\varepsilon}\right\}$,

where $t$ is the 4-momentum-transfer squared at the proton vertex, $\Delta y$ the rapidity-gap width, and $y_{0}$ the center of the rapidity gap. In Eq. (6), the subscript $i=1,2$ enumerates Pomerons in a DPE event, $\Delta y=\Delta y_{1}+\Delta y_{2}$ is the total rapidity gap (sum of two gaps) in the event, and $y_{c}$ is the center in $\eta$ of the centrally-produced hadronic system.

The total cross section $\left(\sigma_{\mathrm{t} t}\right)$ is expressed as:

$\sigma_{\text {tot }}^{p^{ \pm} p}=16.79 s^{0.104}+60.81 s^{-0.32} \mp 31.68 s^{-0.54}$ for $\sqrt{s} \leq 1.8 \mathrm{TeV}, \quad$ (7)

$\sigma_{\text {tot }}^{p^{ \pm} p}=\sigma_{\text {tot }}^{\mathrm{CDF}}+\frac{\pi}{s_{0}}\left[\left(\ln \frac{s}{s_{F}}\right)^{2}-\left(\ln \frac{s^{\mathrm{CDF}}}{s_{F}}\right)^{2}\right]$ for $\sqrt{s} \geq 1.8 \mathrm{TeV}$,

where $s_{0}$ and $s_{F}$ are energy and the Pomeron flux saturation scales, respectively [7]. For $\sqrt{s} \leq 1.8 \mathrm{TeV}$, where there are Reggeon contributions, we use the global fit expression [9], while for $\sqrt{s} \geq 1.8 \mathrm{TeV}$, where Reggeon contributions are negligible, we employ the Froissart-Martin formula [10-12]. The two expressions are smoothly matched at $\sqrt{s} \sim 1.8 \mathrm{TeV}$.

The elastic cross section is obtained from the global fit [9] for $\sqrt{s} \leq 1.8 \mathrm{TeV}$, while for $1.8<\sqrt{s} \leq 50 \mathrm{TeV}$ we use an extrapolation of the global-fit ratio of $\sigma_{\mathrm{el}} / \sigma_{\mathrm{t} t}$, which is slowly varying with $\sqrt{s}$, multiplied by $\sigma_{t o t}$. The total nondiffractive cross section is then calculated as $\sigma_{\mathrm{N} D}=\left(\sigma_{\mathrm{t} o t}-\sigma_{\mathrm{el}}\right)-\left(2 \sigma_{\mathrm{S} D}+\sigma_{\mathrm{D} D}+\sigma_{\mathrm{C} D}\right)$.

\section{RESULTS}

In this section, we present as examples of the predictive power of the RENORM model some results reported by the TOTEM, CMS, and ALICE collaborations for $p p$ collisions at $\sqrt{s}=7 \mathrm{TeV}$, which can be directly compared with RENORM formulas without using the PYTHIA8-MBR simulation.

Fig. (1, left) shows a comparison of the TOTEM total, elastic, and total-inelastic cross sections, along with results from other experiments fitted by the COMPETE Collaboration [13]; the RENORM predictions, displayed as filled (green) squares, are in excellent agreement with the TOTEM results. Similarly, in Fig. (1, right), good agreement is observed between the ALICE [14] and CMS [15] total-inelastic cross sections and the RENORM prediction.

The uncertainty shown in the RENORM prediction of $\sigma_{\text {tot }}$ in Fig. (1, left) is dominated by that in the scale parameter $s_{0}$. The latter can be reduced by a factor of $\sim 4$ if $\sqrt{s_{0}}$ is interpreted as the mean value of the glue-ball-like object discussed in [16] and the data shown in figure 8 of [16] are used to determine its value. Work is in progress to finalize the details of this interpretation.

Another example of the predictive power of RENORM is shown in Fig. (2), which displays the total SD (left) and total DD (right) cross sections for $\xi<0.05$, after extrapolation into the low mass region from the measured CMS cross sections at higher mass regions, presented in [17], using RENORM.

\section{SUMMARY}

We reviewed our pre- LHC predictions for the total, elastic, total-inelastic, and diffractive components of the proton-proton cross section at high energies, which are based on a special parton-model approach to diffraction employing inclusive proton parton distribution functions and QCD color factors. We discuss single diffraction/dissociation, double
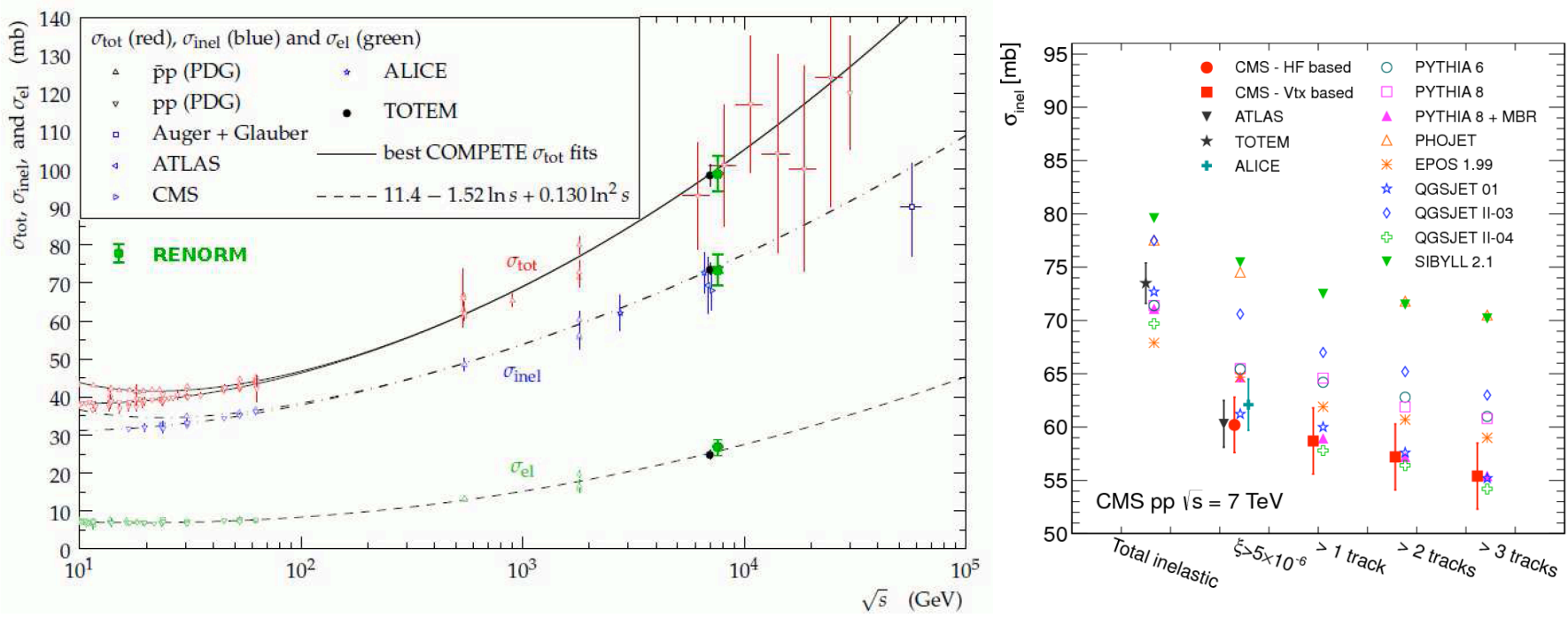

Fig. (1). (left) TOTEM measurements of the total, total-inelastic, and elastic $p p$ cross sections at $\sqrt{s}=7$ TeV shown along with best COMPETE fits [13], with RENORM predictions added as filled squares; (right) ALICE [14] and CMS [15] measurements of the total inelastic cross section at $\sqrt{s}=7 \mathrm{TeV}$ show good agreement with the RENORM prediction (PYTHIA8-MBR). 

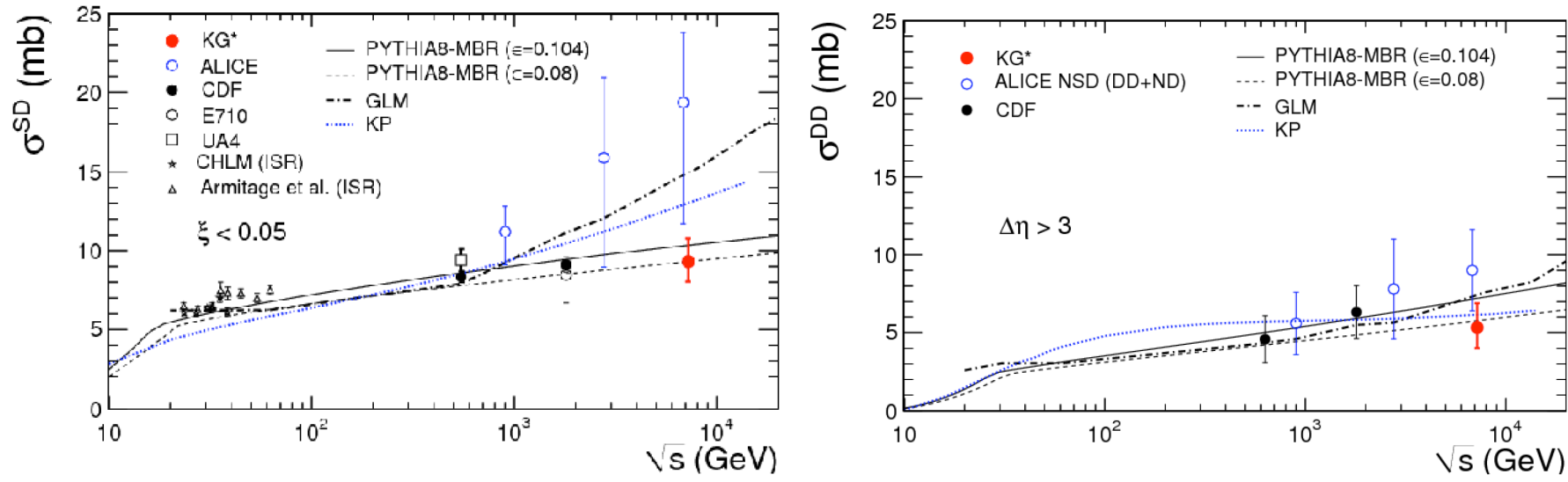

$\mathrm{KG}^{*}$ : obtained by extrapolation of the measured CMS cross sections [17] into the low mass region(s) using the MBR model.

Fig. (2). Measured SD (left) and DD (right) cross sections for $\xi<0.05$ compared with theoretical predictions; the model embedded in PYTHIA8-MBR provides a good description of all data.

diffraction/dissociation, and central diffraction or doublePomeron exchange, comparing predictions with LHC measurements. Agreement between data and PYTHIA8MBR predictions is found in all cases.

\section{CONFLICT OF INTEREST}

The author confirms that this article content has no conflicts of interest.

\section{ACKNOWLEDGEMENTS}

I would like to thank Robert Ciesielski, my colleague at Rockefeller and collaborator in the implementation of the MBR simulation into PYTHIA8, and the Office of Science of the Department of Energy for supporting the Rockefeller experimental diffraction physics programs at Fermilab and LHC on which this research is anchored.

\section{REFERENCES}

[1] Goulianos K. Predictions of Diffractive Cross Sections in ProtonProton Collisions. Proceedings of Diffraction 2012. International Workshop on Diffraction in High Energy Physics; 10-15 September 2012; AIP Conf Proc 2013; 1523: 107-16.

[2] See models presented by various authors in proceedings of Diffraction 2012; AIP Conf Proc 2012; 1523: 107-37.

[3] Goulianos K. Predictions of Diffractive, Elastic, Total, and TotalInelastic pp Cross Sections $v s$ LHC Measurements. Proceedings of XXI International Workshop on Deep-Inelastic Scattering and Related Subject -DIS2013; 22-26 April 2013; Marseilles, France, 2013.

[4] Goulianos K. Hadronic Diffraction: Where do we Stand? Proceedings of Les Rencontres de Physique de la Vallee d'Aoste: Results and Perspectives in Particle Physics, La Thuile, Italy, February 27 - March 6, 2004, Frascati Physics Series, Special 34 Issue, edited by Mario Greco [arXiv:hep-ph/0407035].
[5] Goulianos K. Diffractive and Total pp Cross Sections at LHC. In Mario D, Ed. Proceedings of $13^{\text {th }}$ International Conference on Elastic and Diffractive Scattering (Blois Workshop) - Moving Forward into the LHC Era, CERN, Geneva, Switzerland, June 29July 3, 2009, CERN-Proceedings-2010-02. [arXiv:1002.3527v2].

[6] (a) Sjöstrand T, Mrenna S, Skands P. PYTHIA 6.4 Physics and Manual. J High Energ Phys 2006; 05: 026. (b) A brief introduction to PYTHIA 8.1. Comput Phys Comm 2008; 178: 852.

[7] Ciesielski R, Goulianos K. MBR Monte Carlo Simulation in PYTHIA8. Proc Sci 2013; 301-5.

[8] Goulianos K. A new statistical description of hardonic and $\mathrm{e}^{+} \mathrm{e}-$ multiplicity distributions. Phys Lett B 1987; 193: 151-6.

[9] Covolan RJM, Montanha J, Goulianos K. A new determination of the soft pomeron intercept. Phys Lett B 1996; 389: 176-80.

[10] Froissart M. Asymptotic behavior and subtractions in the mandelstam representation. Phys Rev 1961; 3: 123: 1053-7.

[11] Martin A. Extension of the axiomatic analyticity domain of scattering amplitudes by unitarity, I, II. Nuovo Cimento 1966; 42: 930-53.

[12] Martin A. Froissart bound for inelastic cross sections. Phys Rev D 2009; 80: 065013 .

[13] Deile M (TOTEM Collaboration). Measurements of Proton-Proton and Total Cross Section at the LHC by TOTEM. Proceedings of diffraction 2012 (figure from talk presented at the Conference).

[14] Abelev B, Adam J, Adamová D, et al., ALICE collaboration. Measurement of Inelastic, Single-and Double-Diffraction Cross Sections in Proton-Proton Collisions at the LHC with ALICE, Eur Phys J C 2013; 73:2456. [arXiv:1208.4968].

[15] Chatrchyan S, Khachatryan V, Sirunyan AM, et al. (CMS Collaboration). Measurement of the inelastic proto-proton cross section at $\sqrt{s}=7$ TeV. Phys Lett B 2013; 722: 5 .

[16] Albrow MG, Coughlin TD, Forshaw JR. Central Exclusive Particle Production at High Energy Hadron Colliders. Prog Part Nucl Phys 2010; 65: 149-84.

[17] Ciesielski R (CMS Collaboration). Measurements of diffraction in p-p collisions in CMS: Proceedings of XXI International Workshop on Deep-Inelastic Scattering and Related Subject-DIS2013, pp. 2226 April 2013, Marseilles, France, 2013. 